\title{
CLASSICAL LIMIT FOR SEMI-RELATIVISTIC HARTREE SYSTEMS
}

\author{
GONCA L. AKI, PETER A. MARKOWICH, AND CHRISTOF SPARBER
}

\begin{abstract}
We consider the three-dimensional semi-relativistic Hartree model for fast quantum mechanical particles moving in a self-consistent field. Under appropriate assumptions on the initial density matrix as a (fully) mixed quantum state we prove, using Wigner transformation techniques, that its classical limit yields the well known relativistic Vlasov-Poisson system. The result holds for the case of attractive and repulsive mean-field interaction, with an additional size constraint in the attractive case.
\end{abstract}

\section{INTRODUCTION AND MAIN RESULT}

In this paper we aim to establish the classical limit as $\varepsilon \rightarrow 0_{+}$of the semirelativistic Hartree system (or Schrödinger-Poisson system), i.e.

$$
\left\{\begin{aligned}
i \varepsilon \partial_{t} \rho^{\varepsilon}(t) & =\left[H^{\varepsilon}, \rho^{\varepsilon}(t)\right], \quad x \in \mathbb{R}^{3}, t \in \mathbb{R}_{+}, \\
-\kappa \Delta V^{\varepsilon} & =n^{\varepsilon}(t, x), \quad \kappa= \pm 1
\end{aligned}\right.
$$

subject to an initial data $\rho^{\varepsilon}(0) \equiv \rho_{0}^{\varepsilon}$, where the reason for choosing $\kappa= \pm 1$ respectively will be explained below and the Hamiltonian operator $H^{\varepsilon}$ is given by

$$
H^{\varepsilon}:=\sqrt{-\varepsilon^{2} \Delta+1}+V^{\varepsilon}(t, x) .
$$

Here the pseudo-differential operator for the kinetic energy is simply defined via multiplication in Fourier space with the symbol $\sqrt{|\varepsilon \xi|^{2}+1}$, for the semi-classical parameter $0<\varepsilon \ll 1$, a dimensionless scaled Planck's constant (all other physical constants are rescaled to be equal to 1 ). This scalar pseudo-differential operator is frequently used in relativistic quantum mechanical models as a convenient replacement of the full (matrix-valued) Dirac operator. In (1.1) we denote by $\rho^{\varepsilon}(t) \in \mathfrak{S}_{1}\left(L^{2}\left(\mathbb{R}^{3}\right)\right)$ the density matrix operator of the system, i.e. a positive selfadjoint trace class operator acting on $L^{2}\left(\mathbb{R}^{3}\right)$. The particle density $n^{\varepsilon}(t, \cdot) \in L^{1}\left(\mathbb{R}^{3}\right)$ is then obtained by evaluating the corresponding kernel $\rho^{\varepsilon}(t, x, y)$, which, by abuse of notation is denoted by the same symbol as the density operator, on its diagonal [19, i.e. $n^{\varepsilon}(t, x)=\rho^{\varepsilon}(t, x, x)$. The system (1.1) describes the mean field dynamics of (relativistic) quantum mechanical particles in a mixed state. Denoting by $\left\{\psi_{j}^{\varepsilon}\right\}_{j \in \mathbb{N}}$ a complete orthonormal basis of $L^{2}\left(\mathbb{R}^{3}\right)$ we can decompose the kernel of $\rho^{\varepsilon}(t) \in \mathfrak{S}_{1}\left(L^{2}\left(\mathbb{R}^{3}\right)\right)$ via

$$
\rho^{\varepsilon}(t, x, y)=\sum_{j \in \mathbb{N}} \lambda_{j}^{\varepsilon} \psi_{j}^{\varepsilon}(t, x) \overline{\psi_{j}^{\varepsilon}}(t, y),
$$

2000 Mathematics Subject Classification. 35Q40, 35Q55, 81S30, 81V17.

Key words and phrases. semi-relativistic Hartree energy, classical limit, Wigner transform, relativistic Vlasov-Poisson system.

This work has been supported by the KAUST Investigator Award and the Wolfson Research Merit Award (Royal Society) of P. Markowich. G. L. Aki acknowledges support by the DEASE project of the EU. C. Sparber has been supported by the APART grant of the Austrian Academy of Sciences. 
with (constant in time) $\lambda_{j} \in \ell^{1}, \lambda_{j} \geq 0$ [19]. Using this representation, we arrive at an equivalent system of countable many nonlinear Schrödinger-type equations

$$
\left\{\begin{aligned}
i \varepsilon \partial_{t} \psi_{j}^{\varepsilon} & =\sqrt{-\varepsilon^{2} \Delta+1} \psi_{j}^{\varepsilon}+V^{\varepsilon}(t, x) \psi_{j}^{\varepsilon} \\
-\kappa \Delta V^{\varepsilon} & =n^{\varepsilon}(t, x),
\end{aligned}\right.
$$

where the density is now given by

$$
n^{\varepsilon}(t, x)=\sum_{j \in \mathbb{N}} \lambda_{j}^{\varepsilon}\left|\psi_{j}^{\varepsilon}(t, x)\right|^{2} .
$$

The system (1.2) can be interpreted as the time-dependent model associated to the semi-relativistic Hartree energy

$$
\begin{aligned}
\mathcal{E}_{\mathrm{H}}^{\varepsilon}(t) & =\mathcal{E}_{\text {kin }}^{\varepsilon}(t)+\mathcal{E}_{\text {pot }}^{\varepsilon}(t) \\
& =\sum_{j \in \mathbb{N}} \lambda_{j}^{\varepsilon}\left\|\left(-\varepsilon^{2} \Delta+1\right)^{1 / 4} \psi_{j}^{\varepsilon}\right\|_{L^{2}\left(\mathbb{R}^{3}\right)}^{2}+\frac{\kappa}{8 \pi} \iint_{\mathbb{R}^{6}} \frac{n^{\varepsilon}(t, x) n^{\varepsilon}(t, y)}{|x-y|} \mathrm{d} x \mathrm{~d} y,
\end{aligned}
$$

where for the second line we have used the three dimensional Green's function representation of the potential, i.e.

$$
V^{\varepsilon}(t, x)=\frac{\kappa}{4 \pi|x|} * n^{\varepsilon}(t, x)
$$

For future references we recall that the kinetic and the self-consistent potentialenergy can also be shortly written in terms of density matrices as

$$
\mathcal{E}_{\text {kin }}^{\varepsilon}(t)=\operatorname{tr}\left(\sqrt{-\varepsilon^{2} \Delta+1} \rho^{\varepsilon}(t)\right), \quad \mathcal{E}_{\text {pot }}^{\varepsilon}(t)=\frac{\kappa}{2} \operatorname{tr}\left(V^{\varepsilon}(t, x) \rho^{\varepsilon}(t)\right) .
$$

In the case $\kappa=-1$, the coupling to the Poisson equation comprises an attractive nonlinearity for the Schrödinger-type equations (1.2) and hence, global wellposedness for general initial data does not hold, cf. 5, 13. In this case, the system (1.2) is a generalization (for mixed states) of the semi-relativistic Hartree model derived in 2 as the mean field limit for large systems of Bosons with gravitational self-interaction. This model has been extensively studied in recent years as it is considered to describe the dynamics of so-called Boson stars [4, 5, 6, 13. In the case $\kappa=1$, the Poisson interaction is repulsive and typically models electron-electron self-interactions (in the Hartree-approximation). The system (1.2) therefore allows to study relativistic corrections to the usual Hartree model of many-body electron system as it is needed for example in the case of heavy atoms, cf. [14].

In the present work we are interested in rigorously establishing the classical limit of (1.2) as $\varepsilon \rightarrow 0$. To this end we shall heavily rely on the Wigner transformed picture of quantum mechanics 21. To this end, we define the ( $\varepsilon$-scaled) Wigner transform of a given density matrix kernel $\rho^{\varepsilon}(t, x, y)$ as in [16, 15]

$$
f^{\varepsilon}\left[\rho^{\varepsilon}(t)\right] \equiv f^{\varepsilon}(t, x, \xi):=\frac{1}{(2 \pi)^{3}} \int_{\mathbb{R}^{3}} \rho^{\varepsilon}\left(t, x+\frac{\varepsilon y}{2}, x-\frac{\varepsilon y}{2}\right) e^{-i \xi \cdot y} \mathrm{~d} y,
$$

From this definition we easily infer

$$
\left\|f^{\varepsilon}(t, \cdot, \cdot)\right\|_{L^{2}\left(\mathbb{R}_{x}^{3} \times \mathbb{R}_{\xi}^{3}\right)}^{2}=(2 \pi \varepsilon)^{-3}\left\|\rho^{\varepsilon}(t, \cdot, \cdot)\right\|_{L^{2}\left(\mathbb{R}_{x}^{3} \times \mathbb{R}_{y}^{3}\right)}^{2} .
$$

Since $f^{\varepsilon}(t, x, \xi)$ is real-valued it can be seen as a quantum mechanical analog of a classical phase space distribution. However, the Wigner function in general also takes negative values and therefore does not allow for a probabilistic interpretation. Nevertheless, Wigner functions have proved to be a highly successful tool in the rigorous mathematical derivation of classical limits, see, e.g. [1, 8, 9, 15, 16, 18, 20] for various analytical results and [17] for a numerical study. In particular, under appropriate uniform bounds on $\rho^{\varepsilon}$ (see Theorem 1 below), it is known that $f^{\varepsilon}$ has accumulation points $f \equiv f^{0}(t, x, \xi)$ as $\varepsilon \rightarrow 0$ (in a suitable topology), which are 
positive Borel measures on the phase space. The limiting measures can then be identified as a distributional solution of the corresponding limiting evolutionary system. In our case we expect it to be a solution of the relativistic Vlasov-Poisson system, i.e.

$$
\left\{\begin{array}{l}
\partial_{t} f+\frac{\xi}{\sqrt{|\xi|^{2}+1}} \cdot \nabla_{x} f-\nabla_{x} V \cdot \nabla_{\xi} f=0, \\
-\kappa \Delta V=n(t, x),
\end{array}\right.
$$

where the limiting particle density $n(t, x)$ is obtained as

$$
n(t, x)=\int_{\mathbb{R}^{3}} f(t, x, \xi) \mathrm{d} \xi
$$

The system (1.4) is in itself an intensively studied model, in particular in the gravitational case $\kappa=-1$, see e.g. [10, 11, 12 and the references given therein. The present work thus provides a rigorous connection between the quantum mechanical model (1.1), or equivalently (1.2), and its classical analog (1.4). The novelty of the work lies in the fact that we have to establish suitable estimates based on the relativistic kinetic energy (instead of the usual one $-\frac{\varepsilon^{2}}{2} \Delta$ ) in order to be able to pass to the limit in the nonlinear potential $V^{\varepsilon}(t, x)$. The main result of this paper is as follows.

Theorem 1. Let $\varepsilon_{0}<1$ and assume that the initial data $\rho_{0}^{\varepsilon} \in \mathfrak{S}_{1}\left(L^{2}\left(\mathbb{R}^{3}\right)\right)$ is a density matrix operator, such that

$$
\sup _{\varepsilon \in\left(0, \varepsilon_{0}\right]}\left(\operatorname{tr} \rho_{0}^{\varepsilon}+\frac{1}{\varepsilon^{3}} \operatorname{tr}\left(\rho_{0}^{\varepsilon}\right)^{2}+\operatorname{tr} \sqrt{-\varepsilon^{2} \Delta+1} \rho_{0}^{\varepsilon}\right)<\infty .
$$

In the gravitational case $\kappa=-1$ we additionally assume

$$
\frac{1}{\varepsilon^{3}} \operatorname{tr}\left(\rho_{0}^{\varepsilon}\right)^{2}<\frac{C_{*}}{\operatorname{tr} \rho_{0}^{\varepsilon}}
$$

where $C_{*}>0$ is a fixed $\varepsilon$-independent constant to be computed in the following. Then there exists a unique mild solution $\rho^{\varepsilon} \in C\left([0, \infty) ; \mathfrak{S}_{1}\left(L^{2}\left(\mathbb{R}^{3}\right)\right)\right.$ of the relativistic Hartree system (1.1) and its Wigner transform $f^{\varepsilon}\left[\rho^{\varepsilon}\right] \in C\left([0, \infty) ; L^{2}\left(\mathbb{R}_{x}^{3} \times \mathbb{R}_{\xi}^{3}\right)\right)$ converges, up to extraction of sub-sequences,

$$
f^{\varepsilon}\left[\rho^{\varepsilon}\right] \stackrel{\varepsilon \rightarrow 0}{\longrightarrow} f \quad \text { in } C\left([0, \tau] ; \mathcal{S}^{\prime}\left(\mathbb{R}_{x}^{3} \times \mathbb{R}_{\xi}^{3}\right)-w *\right),
$$

for any $\tau<\infty$, where $f \in C\left([0, \tau] ; \mathcal{M}^{+}\left(\mathbb{R}_{x}^{3} \times \mathbb{R}_{\xi}^{3}\right)\right) \cap L^{\infty}\left([0, \tau] ; L^{1} \cap L^{2}\left(\mathbb{R}_{x}^{3} \times \mathbb{R}_{\xi}^{3}\right)\right)$ is a distributional solution of the relativistic Vlasov-Poisson system (1.4). Here $\mathcal{M}^{+}$ is the space of positive bounded Borel measures on phase-space, equipped with the weak-* topology.

The above given theorem shows that distributional solutions to (1.4) can indeed be interpreted as the classical limit (on any compact time-interval) of solutions to (1.2), or equivalently (1.1). To get more insight on Assumption (A) we again use the decomposition

$$
\rho_{0}^{\varepsilon}=\sum_{j \in \mathbb{N}} \lambda_{j}^{\varepsilon} \psi_{j}^{\varepsilon}(x) \overline{\psi_{j}^{\varepsilon}}(y)
$$

to obtain

$$
\operatorname{tr} \rho_{0}^{\varepsilon}=\sum_{j \in \mathbb{N}} \lambda_{j}^{\varepsilon}, \quad \operatorname{tr}\left(\rho_{0}^{\varepsilon}\right)^{2}=\sum_{j \in \mathbb{N}}\left(\lambda_{j}^{\varepsilon}\right)^{2} .
$$

We remark that $\operatorname{tr} \rho_{0}^{\varepsilon}$ is the total charge or mass of the particle system. Thus Assumption (A) implies a uniform (in $\varepsilon$ ) bound on the total charge/mass and simultaneously requires $\sum_{j \in \mathbb{N}}\left(\lambda_{j}^{\varepsilon}\right)^{2} \simeq \varepsilon^{3}$, as $\varepsilon \rightarrow 0$. (For a construction of a density matrix $\rho_{0}^{\varepsilon}$, which satisfies this requirements for $\psi_{j} \in H^{1}\left(\mathbb{R}^{3}\right)$ we refer to [16].) 
This condition is to be expected from earlier papers [15, 16, and prevents us from establishing our result in the case of pure initial states, i.e. $\rho_{0}^{\varepsilon}=\psi_{j}^{\varepsilon}(x) \overline{\psi_{j}^{\varepsilon}}(y)$, or even finite combinations of pure states. Roughly speaking, the requirement of a totally mixed initial state is needed to ensure that the limiting particle density $n(t, x)$ is not too singular and thus can be successfully convolved with the Poisson kernel $\propto 1 /|x|$. This is not clear a-priori as the limiting $f(t, x, \xi)$, and thus also the limiting density $n(t, x)$, in general is only a measure. In the case of a fully mixed state though we gain a bit more regularity, which is needed in passing to the limit $\varepsilon \rightarrow 0$. Note that for any Hilbert-Schmidt $\rho^{\varepsilon} \in \mathfrak{S}_{2}\left(L^{2}\left(\mathbb{R}^{3}\right)\right)$, it holds

$$
\left\|\rho^{\varepsilon}\right\|_{\mathfrak{S}_{2}\left(L^{2}\left(\mathbb{R}^{3}\right)\right)}^{2}=\operatorname{tr}\left(\rho^{\varepsilon}\right)^{2}=\left\|\rho^{\varepsilon}(\cdot, \cdot)\right\|_{L^{2}\left(\mathbb{R}_{x}^{3} \times \mathbb{R}_{y}^{3}\right)}^{2}<\infty,
$$

cf. [19. Having in mind the scaling property (1.3), Assumption (A) therefore implies for the initial Wigner function $\left\|f^{\varepsilon}(0, \cdot, \cdot)\right\|_{L^{2}}<\infty$, uniformly in $\varepsilon$. This property is shown to be conserved by the time-evolution below and thus, yields an important uniform bound on $f^{\varepsilon}(t, x, \xi)$. Assumption (B) , needed in the gravitational case, then additionally requires that the $L^{2}$-norm of the initial Wigner function or the total charge/mass is sufficiently small (and not only bounded), cf. Remark 2.5 below.

The rest of the paper is now organized as follows: In Section (2) we collect some preliminary results needed for the proof of our main theorem which is given in Section 3. We then close this section with some final remarks on possible generalizations.

\section{Preliminary Results}

Let us start with a technical lemma, that nevertheless turns out to be crucial for the proof of the main Theorem.

Lemma 2.1. Let $\rho(x, y)$ be the kernel of a positive self-adjoint trace class operator $\rho \in \mathfrak{S}_{1}\left(L^{2}\left(\mathbb{R}^{3}\right)\right)$ and $n(x) \equiv \rho(x, x)$ the corresponding density. Then for $p \in[1, \infty)$ the following estimate holds

$$
\|n\|_{L^{q}\left(\mathbb{R}^{3}\right)} \leq C_{p}\|\rho\|_{\mathfrak{S}_{p}\left(L^{2}\left(\mathbb{R}^{3}\right)\right)}^{\theta}(\operatorname{tr}|\nabla| \rho)^{1-\theta},
$$

where

$$
q=\frac{4 p-3}{3 p-2}, \quad \theta=\frac{p}{4 p-3} .
$$

Proof. For the proof we proceed analogously to [15]. Since the case $p=1$ is immediate we consider only $p>1$. We start by considering the operator $|\nabla|-\beta n^{\alpha}$ where $\beta>0$ and $\alpha>0$ are some constants to be chosen later on. Let $\mu_{1} \leq \mu_{2} \leq$ $\ldots$ be the negative eigenvalues of the operator $|\nabla|-\beta n^{\alpha}$ and let $\left\{\varphi_{k}\right\}_{k \in \mathbb{N}}$ be a finite or countable collection of the corresponding ortho-normed eigenvectors. We consequently obtain

$$
\operatorname{tr}\left(|\nabla|-\beta n^{\alpha}\right) \rho \geq \sum_{k \in \mathbb{N}}\left\langle\left(|\nabla|-\beta n^{\alpha}\right) \rho \varphi_{k}, \varphi_{k}\right\rangle=\sum_{j, k \in \mathbb{N}}\left|p_{j k}\right|^{2} \mu_{k} \lambda_{j},
$$

where $p_{j k}=\int_{\mathbb{R}^{3}} \psi_{j} \bar{\varphi}_{k}$. Since $\mu_{k}=-\left|\mu_{k}\right|, \forall k \in \mathbb{N}$, we can rearrange this inequality in the following form

$$
\operatorname{tr} \beta n^{\alpha} \rho=\beta \int_{\mathbb{R}^{3}} n^{\alpha+1} \mathrm{~d} x \leq \operatorname{tr}|\nabla| \rho+\sum_{j, k \in \mathbb{N}}\left|p_{j k}\right|^{2}\left|\mu_{k}\right| \lambda_{j} .
$$


Now, using Hölder's inequality, and the fact that $\sum_{j}\left|p_{j k}\right|^{2}=1$, as well as $\sum_{k}\left|p_{j k}\right|^{2} \leq$ 1 , we obtain

$$
\begin{aligned}
\beta \int_{\mathbb{R}^{3}} n^{\alpha+1} \mathrm{~d} x & \leq \operatorname{tr}|\nabla| \rho+\|\rho\|_{\mathfrak{S}_{p}\left(L^{2}\left(\mathbb{R}^{3}\right)\right)}\left(\sum_{j \in \mathbb{N}}\left|\mu_{j}\right|^{p^{\prime}}\right)^{1 / p^{\prime}} \\
& \leq \operatorname{tr}|\nabla| \rho+\|\rho\|_{\mathfrak{S}_{p}\left(L^{2}\left(\mathbb{R}^{3}\right)\right)} C_{p}\left(\int_{\mathbb{R}^{3}}\left(\beta n^{\alpha}\right)^{3+p^{\prime}} \mathrm{d} x\right)^{1 / p^{\prime}} .
\end{aligned}
$$

Here the second inequality is obtained from Theorem 2.1 in [3], which states that for all $\delta>0$

$$
\sum_{j \geq 1}\left|\mu_{j}\right|^{\delta} \leq C_{\delta, d} \int_{\mathbb{R}^{d}} W(x)_{+}^{\delta+d} \mathrm{~d} x
$$

where $\mu_{j}$ are the negative eigenvalues of the operator $|\nabla|-W$. Now with the choice $\alpha=(p-1) /(3 p-2)$, setting $\alpha+1=q$ and

$$
\beta=\left(\frac{\left(\int_{\mathbb{R}^{3}} n^{q} \mathrm{~d} x\right)^{1 / p}}{2 C\|\rho\|_{\mathfrak{S}_{p}\left(L^{2}\left(\mathbb{R}^{3}\right)\right)}}\right)^{p^{\prime} / 3},
$$

it is easy to conclude

$$
\left(\int_{\mathbb{R}^{3}} n^{q} \mathrm{~d} x\right)^{1 / q} \leq C_{p}\left\|\rho^{\varepsilon}\right\|_{\mathfrak{S}_{p}\left(L^{2}\left(\mathbb{R}^{3}\right)\right)}^{\theta}(\operatorname{tr}|\nabla| \rho)^{1-\theta} .
$$

Remark 2.2. The proof can easily be generalized to the $d$-dimensional case, where one finds

$$
q=\frac{d(p-1)+p}{d(p-1)+1}, \quad \theta=\frac{p}{d(p-1)+p}
$$

Next, we state a local-in-time existence result for the Hartree system (1.2).

Lemma 2.3. Let $\rho_{0}^{\varepsilon}$ be such that Assumption (A) holds. Then there exists a $T>0$ and unique mild solution $\rho^{\varepsilon} \in C\left([0, T) ; \mathfrak{S}_{1}\left(L^{2}\left(\mathbb{R}^{3}\right)\right)\right.$ to (1.2), or equivalently (1.1), which satisfies the following conservation laws:

$$
\operatorname{tr} \rho^{\varepsilon}(t)=\operatorname{tr} \rho_{0}^{\varepsilon}, \quad \operatorname{tr}\left(\rho^{\varepsilon}(t)\right)^{2}=\operatorname{tr}\left(\rho_{0}^{\varepsilon}\right)^{2} .
$$

Proof. The existence of a unique solution can be obtained in a straightforward way from the results given in [5] (where the case of density matrices with finite rank is treated). Also, the conservation laws (2.2) are established there.

Note that the conservation laws (2.2) together with Assumption (A) directly imply that, for all $\varepsilon \in\left(0, \varepsilon_{0}\right]$, it holds

$$
\left\|n^{\varepsilon}(t)\right\|_{L^{1}\left(\mathbb{R}^{3}\right)}=\left\|n^{\varepsilon}(0)\right\|_{L^{1}\left(\mathbb{R}^{3}\right)}<\infty,
$$

and

for all $t \in[0, T)$.

$$
\left\|f^{\varepsilon}(t)\right\|_{L^{2}\left(\mathbb{R}_{x}^{3} \times \mathbb{R}_{\xi}^{3}\right)}=\left\|f^{\varepsilon}(0)\right\|_{L^{2}\left(\mathbb{R}_{x}^{3} \times \mathbb{R}_{\xi}^{3}\right)}<\infty
$$

Lemma 2.4. The solution stated above exists globally in-time, i.e. $T=\infty$, and we additionally have conservation of energy: $\mathcal{E}_{\mathrm{H}}^{\varepsilon}(t)=\mathcal{E}_{\mathrm{H}}^{\varepsilon}(0)$, provided that one of the following conditions is satisfied:

i) $\kappa=1$,

ii) $\kappa=-1$ and Assumption (B) is satisfied. 
In particular we have the following uniform bound

$$
\mathcal{E}_{\text {kin }}^{\varepsilon}(t) \leq C, \quad \forall t \in[0, \infty) .
$$

Proof. Formally, the conservation of energy can be obtained as in 5 . In order to obtain the uniform a-priori bound (2.3) we shall use (2.1), with $p=2$, which yields

$$
\begin{aligned}
\left\|n^{\varepsilon}(t)\right\|_{L^{5 / 4}} & \leq C_{2}\left\|\rho^{\varepsilon}(t, \cdot, \cdot)\right\|_{L^{2}}^{2 / 5}\left(\operatorname{tr}|\nabla| \rho^{\varepsilon}(t)\right)^{3 / 5} \\
& \leq C_{2} \varepsilon^{-3 / 5}\left\|\rho^{\varepsilon}(0, \cdot, \cdot)\right\|_{L^{2}}^{2 / 5}\left(\operatorname{tr} \sqrt{-\varepsilon^{2} \Delta+1} \rho^{\varepsilon}(t)\right)^{3 / 5}
\end{aligned}
$$

due to the second conservation law in (2.2) and the fact that $\varepsilon|\nabla| \leq \sqrt{-\varepsilon^{2} \Delta+1}$, as can be seen on the level of the Fourier-symbols. On the other hand, recalling the Sobolev inequality

$$
\iint_{\mathbb{R}^{3} \times \mathbb{R}^{3}} \frac{n^{\varepsilon}(t, x) n^{\varepsilon}(t, y)}{|x-y|} \mathrm{d} x \mathrm{~d} y \leq C_{\mathrm{s}}\left\|n^{\varepsilon}(t)\right\|_{L^{6 / 5}}^{2},
$$

and interpolating the density via $\left\|n^{\varepsilon}(t)\right\|_{L^{6 / 5}} \leq\left\|n^{\varepsilon}(t)\right\|_{L^{1}}^{1 / 6}\left\|n^{\varepsilon}(t)\right\|_{L^{5 / 4}}^{5 / 6}$, we obtain

$$
\iint_{\mathbb{R}^{3} \times \mathbb{R}^{3}} \frac{n^{\varepsilon}(t, x) n^{\varepsilon}(t, y)}{|x-y|} \mathrm{d} x \mathrm{~d} y \leq \tilde{C} \mathcal{E}_{\text {kin }}^{\varepsilon}(t), \quad \forall t \in[0, \infty),
$$

where $\tilde{C}=C_{\mathrm{s}} C_{2}^{5 / 3}\left(\operatorname{tr} \rho_{0}^{\varepsilon}\right)^{1 / 3}\left\|f^{\varepsilon}(0, \cdot, \cdot)\right\|_{L^{2}}^{2 / 3}$ is $\varepsilon$-independent by Assumption (A). On the one hand, this shows that the initial energy is indeed well defined and uniformly bounded in $\varepsilon$ for both $\kappa= \pm 1$. Moreover, we immediately conclude the uniform boundedness of the kinetic energy in the case $\kappa=1$ and hence, global-in-time existence of solutions. In the case $\kappa=-1$ we only get, from energy conservation, that

$$
\mathcal{E}_{\mathrm{H}}^{\varepsilon}(0)=\mathcal{E}_{\text {kin }}^{\varepsilon}(t)-\frac{1}{8 \pi} \iint_{\mathbb{R}^{3} \times \mathbb{R}^{3}} \frac{n^{\varepsilon}(t, x) n^{\varepsilon}(t, y)}{|x-y|} \mathrm{d} x \mathrm{~d} y \geq\left(1-\frac{\tilde{C}}{8 \pi}\right) \mathcal{E}_{\mathrm{kin}}^{\varepsilon}(t) .
$$

Uniform boundedness of $\mathcal{E}_{\text {kin }}^{\varepsilon}(t)$, and hence, global-in-time existence, can therefore be concluded if $\tilde{C}<8 \pi$, which holds true under Assumption B.

Remark 2.5. In comparison to the proof given in 13 we needed to invoke slightly different arguments in order to obtain the uniform (w.r.t. $\varepsilon$ ) bound (2.3). The requirement $\tilde{C}<8 \pi$ directly leads to Assumption (B) with $C_{*}=\frac{(8 \pi)^{3}}{C_{\mathrm{s}}^{3} C_{2}^{5}}$. Note however, that in our mixed-state case we can no longer characterize the condition $\tilde{C}<8 \pi$ by the solution of the corresponding single-state ground state problem as it is done in 13 .

\section{Proof of the Main Result}

In this paper we shall use the following definition for the Fourier transform

$$
\left(\mathcal{F}_{\xi \rightarrow \eta} \varphi\right)(\eta) \equiv \hat{\varphi}(\eta)=\int_{\mathbb{R}^{3}} \varphi(\xi) e^{-i \xi \cdot \eta} \mathrm{d} \xi
$$

Moreover we denote by $\mathcal{S}$ the Schwartz space of rapidly decaying functions.

Proof. The proof of the Theorem 1 consists of three steps:

Step 1. We first note that due to Assumption (A) and the conservation laws (2.2), the Wigner function $f^{\varepsilon}(t)$ is uniformly bounded in $L^{2}\left(\mathbb{R}_{x}^{3} \times \mathbb{R}_{\xi}^{3}\right)$ and in $\mathcal{S}^{\prime}\left(\mathbb{R}_{x}^{3} \times \mathbb{R}_{\xi}^{3}\right)$, where the later follows from the results in [9]. Thus, for every fixed $t \in[0, \infty)$, it holds (up to extraction of sub-sequences): $f^{\varepsilon}(t) \rightarrow f(t)$, as $\varepsilon \rightarrow 0$, in $\mathcal{S}\left(\mathbb{R}_{x}^{3} \times \mathbb{R}_{\xi}^{3}\right)-w *$ and in $L^{2}\left(\mathbb{R}_{x}^{3} \times \mathbb{R}_{\xi}^{3}\right)-w$. Moreover it has been shown in [9, 15] 
that the limit $f \in \mathcal{M}^{+}\left(\mathbb{R}_{x}^{3} \times \mathbb{R}_{\xi}^{3}\right)$, i.e. a positive phase-space measure.

Step 2. Next we shall prove the time-equicontinuity of $f^{\varepsilon}(t, x, \xi)$. To this end, we have to show that $\partial_{t} f^{\varepsilon}$ is bounded in $L^{\infty}\left((0, \tau), \mathcal{S}^{\prime}\left(\mathbb{R}_{x}^{3} \times \mathbb{R}_{\xi}^{3}\right)\right)$, for any $\tau<$ $\infty$. We consequently consider the (phase space) weak formulation of the Wigner transformed evolution equation, i.e.

$$
-\left\langle\partial_{t} f^{\varepsilon}, \phi\right\rangle=\left\langle\Gamma^{\varepsilon} f^{\varepsilon}, \phi\right\rangle+\left\langle\Theta^{\varepsilon}\left[V^{\varepsilon}\right] f^{\varepsilon}, \phi\right\rangle,
$$

where $\phi \in \mathcal{S}\left(\mathbb{R}_{x}^{3} \times \mathbb{R}_{\xi}^{3}\right)$ and we write $\langle\cdot, \cdot\rangle$ for the corresponding duality bracket on $\mathcal{S}$. Above, $\Gamma^{\varepsilon}$ and $\Theta^{\varepsilon}\left[V^{\varepsilon}\right]$ are pseudo-differential operators, corresponding to the Wigner transformed kinetic and potential energy terms. Explicitly, $\Gamma^{\varepsilon} f^{\varepsilon}$ is given by

$$
\Gamma^{\varepsilon} f^{\varepsilon}(t, x, \xi)=\frac{i}{(2 \pi)^{3}} \iint_{\mathbb{R}^{3} \times \mathbb{R}^{3}} e^{i y \cdot(x-\eta)} y \cdot \gamma^{\varepsilon}(y, \xi) f^{\varepsilon}(t, \eta, \xi) \mathrm{d} y \mathrm{~d} \eta,
$$

where

$$
\gamma^{\varepsilon}(y, \xi):=\frac{2 \xi}{\sqrt{\left|\xi+\varepsilon \frac{y}{2}\right|^{2}+1}+\sqrt{\left|\xi-\varepsilon \frac{y}{2}\right|^{2}+1}} \in \mathbb{R}^{3} .
$$

On the other hand, the nonlinear potential $V^{\varepsilon}(t, x)$ enters via the well known operator, cf. [15, 16]

$$
\Theta^{\varepsilon}\left[V^{\varepsilon}\right] f^{\varepsilon}(t, x, \xi)=\frac{i}{(2 \pi)^{3}} \iint_{\mathbb{R}^{3} \times \mathbb{R}^{3}} e^{i \eta \cdot(z-\xi)} \delta^{\varepsilon}(t, x, \eta) f^{\varepsilon}(t, x, z) \mathrm{d} \eta \mathrm{d} z .
$$

whose symbol is given by

$$
\delta^{\varepsilon}(t, x, \eta):=\frac{1}{\varepsilon}\left(V^{\varepsilon}\left(t, x+\varepsilon \frac{\eta}{2}\right)-V^{\varepsilon}\left(t, x-\varepsilon \frac{\eta}{2}\right)\right)=\eta \cdot \int_{-1 / 2}^{1 / 2} \nabla_{x} V^{\varepsilon}(t, x+\varepsilon s \eta) \mathrm{d} s .
$$

In order to estimate the first term on the r.h.s. of (3.1), we use an inverse Fourier transform w.r.t. $x$ and Plancherel's theorem to obtain

$$
\begin{aligned}
\left|\left\langle\Gamma^{\varepsilon} f^{\varepsilon}, \phi\right\rangle\right| & =\left|\iint_{\mathbb{R}^{6}}\left(\int_{\mathbb{R}^{3}} e^{-i y \cdot \eta}\left(\mathcal{F}_{x \rightarrow y}^{-1} \nabla_{x} \phi\right)(y, \xi) \cdot \gamma^{\varepsilon}(\xi, y) \mathrm{d} y\right) f^{\varepsilon}(t, \eta, \xi) \mathrm{d} \eta \mathrm{d} \xi\right| \\
& \leq\left\|f^{\varepsilon}(t)\right\|_{L^{2}}\left(\iint_{\mathbb{R}^{6}}\left|\left(\mathcal{F}_{x \rightarrow y}^{-1} \nabla_{x} \phi\right)(y, \xi)\right|^{2}\left|\gamma^{\varepsilon}(\xi, y)\right|^{2} \mathrm{~d} y \mathrm{~d} \xi\right)^{1 / 2} \\
& \leq\left\|f^{\varepsilon}(t)\right\|_{L^{2}}\left\|\nabla_{x} \phi\right\|_{L^{2}},
\end{aligned}
$$

where the second inequality follows from the fact that $\left|\gamma^{\varepsilon}\right| \leq 1$, for all $\varepsilon \in\left(0, \varepsilon_{0}\right]$. Since $f^{\varepsilon}(t, x, \xi)$ is uniformly bounded in $L^{2}\left(\mathbb{R}_{x}^{3} \times \mathbb{R}_{\xi}^{3}\right)$ for all $t \in[0, \infty)$, due to (2.2) and Assumption (A) , we obtain the desired uniform bound for $\left|\left\langle\Gamma^{\varepsilon} f^{\varepsilon}, \phi\right\rangle\right|$. Next, we consider the nonlinear term on the r.h.s. of (3.1). After a Fourier transform w.r.t. $\xi$, we need to estimate

$$
\left|\left\langle\Theta^{\varepsilon}\left[V^{\varepsilon}\right] f^{\varepsilon}, \phi\right\rangle\right|=\left|\iint_{\mathbb{R}^{6}} \hat{\phi}(x, \eta)\left(\int_{-1 / 2}^{1 / 2} \eta \cdot \nabla_{x} V^{\varepsilon}(t, x+\varepsilon s \eta) \mathrm{d} s\right)\left(\mathcal{F}_{x \rightarrow y}^{-1} f^{\varepsilon}\right)(t, x, \eta) \mathrm{d} x \mathrm{~d} \eta\right| .
$$

Using the generalized Young inequality we have

$$
\left\|\nabla_{x} V^{\varepsilon}(t)\right\|_{L^{2}\left(\mathbb{R}^{3}\right)} \leq C\left\|\nabla_{x}|x|^{-1}\right\|_{L_{w}^{3 / 2}\left(\mathbb{R}^{3}\right)}\left\|n^{\varepsilon}(t)\right\|_{L^{6 / 5}\left(\mathbb{R}^{3}\right)},
$$

and by interpolation between $L^{1}\left(\mathbb{R}^{3}\right)$ and $L^{5 / 4}\left(\mathbb{R}^{3}\right)$, one obtains

$$
\left\|n^{\varepsilon}(t)\right\|_{L^{6 / 5}} \leq\left\|n^{\varepsilon}(t)\right\|_{L^{1}}^{1 / 6}\left\|n^{\varepsilon}(t)\right\|_{L^{5 / 4}}^{5 / 6} \leq \operatorname{tr}\left(\rho^{\varepsilon}(t)\right)^{1 / 6}\left\|f(t, \cdot, \cdot)^{\varepsilon}\right\|_{L^{2}}^{1 / 3}\left(\mathcal{E}_{\text {kin }}^{\varepsilon}(t)\right)^{1 / 2} .
$$

Due to the conservation laws (2.2) and the uniform bound on the kinetic energy (2.3) we therefore find that $\left\|\nabla_{x} V^{\varepsilon}(t)\right\|_{L^{2}}$ is uniformly bounded as $\varepsilon \rightarrow 0$, for all 
$t \in[0, \infty)$. Thus, we can estimate

$$
\begin{aligned}
\left|\left\langle\Theta^{\varepsilon}\left[V^{\varepsilon}\right] f^{\varepsilon}, \phi\right\rangle\right| & \leq\left\|f^{\varepsilon}(t)\right\|_{L^{2}}\left(\iint_{\mathbb{R}^{6}}|\hat{\phi}(x, \eta)|^{2}|\eta|^{2}\left|\int_{-1 / 2}^{1 / 2} \nabla_{x} V^{\varepsilon}(t, x+\varepsilon s \eta) \mathrm{d} s\right|^{2} \mathrm{~d} x \mathrm{~d} \eta\right)^{1 / 2} \\
& \leq\left\|f^{\varepsilon}(t)\right\|_{L^{2}}\left\|\nabla_{x} V^{\varepsilon}(t)\right\|_{L^{2}}\left(\int_{\mathbb{R}^{3}} \sup _{y \in \mathbb{R}^{3}}|\hat{\phi}(y-\varepsilon s \eta, \eta)|^{2}|\eta|^{2} \mathrm{~d} \eta\right)^{1 / 2} \\
& \leq\left\|f^{\varepsilon}(t)\right\|_{L^{2}}\left\|\nabla_{x} V^{\varepsilon}(t)\right\|_{L^{2}}\left\|\operatorname { s u p } _ { y \in \mathbb { R } ^ { 3 } } \left|\hat{\phi}(y, \eta)\|\eta \mid\|_{L^{2}}<\infty,\right.\right.
\end{aligned}
$$

uniformly in $\varepsilon$. Thus, $\partial_{t} f^{\varepsilon}$ is bounded in $L^{\infty}\left((0, \tau), \mathcal{S}^{\prime}\left(\mathbb{R}_{x}^{3} \times \mathbb{R}_{\xi}^{3}\right)\right)$, uniformly in $\varepsilon$, and we conclude that (again up to extraction of sub-sequences)

$$
\begin{array}{rll}
f^{\varepsilon \stackrel{\varepsilon \rightarrow 0}{\longrightarrow} f} \quad \text { in } & C\left((0, \tau) ; \mathcal{S}^{\prime}\left(\mathbb{R}_{x}^{3} \times \mathbb{R}_{\xi}^{3}\right)-w *\right), \\
\text { and } \quad \text { in } & L^{\infty}\left((0, \tau) ; L^{2}\left(\mathbb{R}_{x}^{3} \times \mathbb{R}_{\xi}^{3}\right)\right)-w * .
\end{array}
$$

Together with the results of Step 1 this implies

$$
f \in C\left((0, \tau) ; \mathcal{M}^{+}\left(\mathbb{R}_{x}^{3} \times \mathbb{R}_{\xi}^{3}\right)-w *\right) \cap L^{\infty}\left((0, \tau) ; L^{1} \cap L^{2}\left(\mathbb{R}_{x}^{3} \times \mathbb{R}_{\xi}^{3}\right)\right) .
$$

Step 3. It remains to identify the limiting $f(t, x, \xi)$, by passing to the limit in the (full) weak formulation of the Wigner transformed evolution equation, i.e.

$$
\begin{aligned}
& \int_{0}^{\infty} \iint_{\mathbb{R}^{6}}\left(-\partial_{t} \sigma(t) \phi(x, \xi)-\sigma(t) \Gamma^{\varepsilon} \phi(x, \xi)\right) f^{\varepsilon}(t, x, \xi) \mathrm{d} x \mathrm{~d} \xi \mathrm{d} t \\
& +\int_{0}^{\infty} \iint_{\mathbb{R}^{6}} \sigma(t) \Theta^{\varepsilon}\left[V^{\varepsilon}\right] \phi(x, \xi) f^{\varepsilon}(t, x, \xi) \mathrm{d} x \mathrm{~d} \xi \mathrm{d} t=\iint_{\mathbb{R}^{6}} \sigma(0) \phi(x, \xi) f_{0}^{\varepsilon}(x, \xi) \mathrm{d} x \mathrm{~d} \xi
\end{aligned}
$$

where $\sigma \in C_{0}^{\infty}\left(\mathbb{R}_{t}^{+}\right)$and $\phi \in \mathcal{S}\left(\mathbb{R}_{x}^{3} \times \mathbb{R}_{\xi}^{3}\right)$. First it is easily seen that

$$
\lim _{\varepsilon \rightarrow 0} \sup _{t \in[0, \tau]}\left|\left\langle f^{\varepsilon}, \Gamma^{\varepsilon} \phi\right\rangle-\left\langle f, \nabla_{x} \phi \cdot \frac{\xi}{\sqrt{|\xi|^{2}+1}}\right\rangle\right|=0,
$$

since $f^{\varepsilon} \rightarrow f$ in $L^{\infty}\left((0, \tau] ; L^{2}\left(\mathbb{R}_{x}^{3} \times \mathbb{R}_{\xi}^{3}\right)\right)-w *$, as shown in Step 2, and the convergence of the rest of the duality bracket is strong in $C\left([0, T] ; L^{2}\left(\mathbb{R}_{x}^{3} \times \mathbb{R}_{\xi}^{3}\right)\right)$. Concerning the nonlinear term, we have to show

$$
\lim _{\varepsilon \rightarrow 0} \sup _{t \in[0, \tau]}\left|\left\langle f^{\varepsilon}, \Theta^{\varepsilon}\left[V^{\varepsilon}\right] \phi\right\rangle-\left\langle f, \nabla_{x} V \cdot \nabla_{\xi} \phi\right\rangle\right|=0 .
$$

Similarly to what is done above, after invoking a Fourier transform w.r.t. $\xi$, it is sufficient to prove that

$$
\lim _{\varepsilon \rightarrow 0}\left(\hat{\phi}(x, \eta) \int_{-1 / 2}^{1 / 2} \eta \cdot \nabla_{x} V^{\varepsilon}(t, x+\varepsilon s \eta) \mathrm{d} s\right)=\hat{\phi}(x, \eta) \eta \cdot \nabla_{x} V(t, x),
$$

in $C\left([0, T] ; L^{2}\left(\mathbb{R}_{x}^{3} \times \mathbb{R}_{\xi}^{3}\right)\right)$ strongly. In order to do so we denote the compact support of $\hat{\phi}$ by $\Omega: \subseteq \Omega_{x} \times \Omega_{\eta}$ and write

$$
\begin{aligned}
& \int_{-1 / 2}^{1 / 2}\left(\left.\iint_{\Omega}|\hat{\phi}(x, \eta)| \eta\right|^{2}\left|\nabla_{x} V^{\varepsilon}(t, x+\varepsilon s \eta)-\nabla_{x} V(t, x)\right|^{2} \mathrm{~d} x \mathrm{~d} \eta\right)^{1 / 2} \mathrm{~d} s \\
& \leq \sup _{x, \eta \in \Omega}|\hat{\phi}(x, \eta)| \eta|| \int_{-1 / 2}^{1 / 2}\left(\iint_{\Omega}\left|\nabla_{x} V^{\varepsilon}(t, x+\varepsilon s \eta)-\nabla_{x} V(t, x+\varepsilon s \eta)\right|^{2} \mathrm{~d} x \mathrm{~d} \eta\right. \\
& \left.\quad+\iint_{\Omega}\left|\nabla_{x} V(t, x+\varepsilon s \eta)-\nabla_{x} V(t, x)\right|^{2} \mathrm{~d} x \mathrm{~d} \eta\right)^{1 / 2} \mathrm{~d} s \\
& =: \sup _{x, \eta \in \Omega}|\hat{\phi}(x, \eta)| \eta|| \int_{-1 / 2}^{1 / 2}\left(I^{\varepsilon}+J^{\varepsilon}\right)^{1 / 2} \mathrm{~d} s .
\end{aligned}
$$


First, we consider the term $J^{\varepsilon}$ : Using Plancherel's Theorem we can write

$$
\begin{aligned}
J^{\varepsilon} & =\iint_{\Omega}\left|\nabla_{x} V(t, x+\varepsilon s \eta)-\nabla_{x} V(t, x)\right|^{2} \mathrm{~d} x \mathrm{~d} \eta \\
& =\iint_{\Omega}\left|\left(\mathcal{F}_{x \rightarrow z} \nabla_{x} V\right)(t, z)\right|^{2}\left|e^{i \varepsilon s \eta \cdot z}-1\right|^{2} \mathrm{~d} x \mathrm{~d} \eta,
\end{aligned}
$$

from which we conclude that $J^{\varepsilon} \rightarrow 0$, as $\varepsilon \rightarrow 0$, by dominant convergence. In order to treat the term $I^{\varepsilon}$ we take into account that $V^{\varepsilon}$ solves the Poisson equation

$$
-\kappa \Delta V^{\varepsilon}=n^{\varepsilon}(t, x), \quad \kappa= \pm 1
$$

with $n^{\varepsilon}(t) \in L^{5 / 4}\left(\mathbb{R}^{3}\right) \cap L^{1}\left(\mathbb{R}^{3}\right)$, uniformly in $\varepsilon$. Due to the regularizing property of the Poisson equation we have $\nabla_{x} V^{\varepsilon} \in W_{\text {loc }}^{1,5 / 4}\left(\mathbb{R}^{3}\right)$ uniformly in $\varepsilon$, which is compactly embedded in $L_{\text {loc }}^{2}\left(\mathbb{R}^{3}\right)$. We therefore infer that, for all $t \in[0, \tau]$, it holds

$$
\nabla_{x} V^{\varepsilon}(t) \stackrel{\varepsilon \rightarrow 0}{\longrightarrow} \nabla_{x} V(t) \quad \text { in } \quad L^{2}(\mathcal{O}),
$$

where $\mathcal{O}=\left\{z:=x+\varepsilon s \eta \in \mathbb{R}^{3} \mid(x, \eta) \in\left(\Omega_{x}^{3} \times \Omega_{\eta}^{3}\right)\right\}$. We finally recall the result of Theorem III.2 in 15 , which (up to extraction of subsequences) states that, as $\varepsilon \rightarrow 0$,

$$
n^{\varepsilon}(t, x) \rightarrow n(t, x)=\int_{\mathbb{R}^{3}} f(t, x, \xi) \mathrm{d} \xi,
$$

in $C\left([0, T] ; \mathcal{M}^{+}\left(\mathbb{R}_{x}^{3}\right)-w *\right)$. We therefore conclude $I^{\varepsilon} \rightarrow 0$, as $\varepsilon \rightarrow 0$, which consequently implies (3.3). In summary we have shown that the Wigner transformed evolution equation (3.2) converges weakly to

$$
\begin{aligned}
& \int_{0}^{\infty} \iint_{\mathbb{R}^{6}}\left(-\partial_{t} \sigma(t) \phi(x, \xi)-\frac{\xi}{\sqrt{|\xi|^{2}+1}} \cdot \nabla_{x} \phi(x, \xi) \sigma(t)\right) f(t, x, \xi) \mathrm{d} x \mathrm{~d} \xi \mathrm{d} t \\
& +\int_{0}^{\infty} \iint_{\mathbb{R}^{6}} \sigma(t) \nabla_{x} V \cdot \nabla_{\xi} \phi(x, \xi) f(t, x, \xi) \mathrm{d} x \mathrm{~d} \xi \mathrm{d} t=\iint_{\mathbb{R}^{6}} \sigma(0) \phi(x, \xi) f_{0}(x, \xi) \mathrm{d} x \mathrm{~d} \xi
\end{aligned}
$$

i.e. the relativistic Vlasov-Poisson system (in distributional sense). This finishes the proof.

Remark 3.1. It is straightforward to generalize our result for Yukawa-type interactions, i.e.

$$
V^{\varepsilon}(t, x)=\kappa \frac{e^{-\lambda|x|}}{4 \pi|x|} * n^{\varepsilon}(t, x), \quad \lambda>0,
$$

which are used in, e.g. 13. Indeed it is possible to prove our theorem for an even more general class of self-consistent interaction potentials $V^{\varepsilon}=G * n^{\varepsilon}$, if the kernel $G=G(x)$ satisfies appropriate regularity conditions, cf. [1, 15 for a discussion in the case of non-relativistic Hartree systems. Finally, we remark that one can also treat the case of semi-relativistic Hartree-Fock systems, cf. [5], by combining straightforwardly the results present here with those given in [7] for non-relativistic Hartree-Fock systems. To this end, one should note that the so-called exchangeterm vanishes as $\varepsilon \rightarrow 0$. Hence, one again recovers the relativistic Vlasov-Poisson system (1.4) in the limit.

\section{REFERENCES}

1. R. Bosi, Classical limit for linear and nonlinear quantum Fokker-Planck systems, preprint 2007.

2. A. Elgart and B. Schlein, Mean Field Dynamics of Boson Stars, Comm. Pure and Appl. Math. 60 (2007), no. 4, 500-545.

3. R. L. Frank, E. H. Lieb, and R. Seiringer, Hardy-Lieb-Thirring inequalities for fractional Schrödinger operators, J. Amer. Math. Soc. 21 (2008), 925-950.

4. J. Fröhlich, B. L. Jonsson, and E. Lenzmann, Effective dynamics for boson stars, Nonlinearity 20 (2007), no. 5, 1031-1075. 
5. J. Fröhlich, and E. Lenzmann, Dynamical Collapse of White Dwarfs in Hartree- and HartreeFock Theory, Comm. Math. Phys. 274 (2007), no. 3, 737-750.

6. J. Fröhlich and E. Lenzmann, Blowup for nonlinear wave equations describing boson stars, Comm. Pure Appl. Math. 60 (2007), no. 11, 1691-1705.

7. I. Gasser, R. Illner, P. Markowich, and C. Schmeiser, Semiclassical, $t \rightarrow \infty$ asymptotics and dispersive Effects for Hartree-Fock Systems, Math. Model. Num. Anal. 32 (1998), 699-713.

8. I. Gasser, C. K. Lin, P. A. Markowich, A review of dispersive limits of (non)linear Schrödinger-type equations, Taiwanese J. Math. 4 (2000), 501-529.

9. P. Gérard, P. Markowich, N. Mauser, and F. Poupaud, Homogenization Limits and Wigner transforms, Comm. Pure Appl. Math. 50 (1997), 323-379.

10. R. Glassey and J. Schaeffer, On symmetric solutions of the relativistic Vlasov-Poisson system, Comm. Math. Phys. 101 (1985), no. 4, 459-473.

11. R. Glassey and J. Schaeffer, On global symmetric solutions to the relativistic Vlasov-Poisson equation in three space dimensions, Math. Methods Appl. Sci. 24 (2001), no. 3, 143-157.

12. M. Hadzic and G. Rein, Global existence and nonlinear stability for the relativistic VlasovPoisson system in the gravitational case, Indiana Univ. Math. J. 56 (2007), 2453-2488.

13. E. Lenzmann, Well-Posedness for Semi-Relativistic Hartree Equations of Critical Type, Math. Phys. Anal. Geom. 10 (2007), no. 1, 43-64.

14. E. Lieb, The stability of matter: From atoms to stars, Selecta of Elliot H. Lieb (W. Thirring, ed.), Springer 1991.

15. P.-L. Lions and T. Paul, Sur les measures de Wigner, Rev. Math. Iberoamericana 9 (1993), 553-618.

16. P. Markowich and N. Mauser, The classical limit of a self-consistent quantum-Vlasov equation in 3-D, Math. Models Methods Appl. Sci. 3 (1993), no. 1, 109-124.

17. P. Markowich, P. Pietra, C. Pohl, Numerical approximation of quadratic observables of Schrödinger-type equations in the semi-classical limit, Numer. Math. 81 (1999), 595-630.

18. M. Pulvirenti, Semiclassical expansion of Wigner functions, J. Math. Phys. 47 (2006), 5210352114.

19. B. Simon, Trace ideals and their applications, Cambridge Univ. Press (1979).

20. C. Sparber, P. A. Markowich and N. J. Mauser, Wigner functions versus WKB-methods in multivalued geometrical optics, Asymp. Anal. 33 (2003) 153-187.

21. E. Wigner, On the quantum correction for the thermodynamical equilibrium, Phys. Rev. 40 (1932), 742-759.

(G. L. Aki) Faculty of Mathematics, University of Vienna, Nordbergstrasse 15, A1090 Vienna, Austria

E-mail address: gonca.aki@univie.ac.at

(P. A. Markowich) Department of Applied Mathematics and Theoretical Physics, University of Cambridge, Wilberforce Road, Cambridge CB3 0WA \& Faculty of Mathematics, University of Vienna, Nordbergstrasse 15, A-1090 Vienna, Austria

E-mail address: peter.markowich@univie.ac.at

(C. Sparber) Department of Applied Mathematics and Theoretical Physics, University of Cambridge, Wilberforce Road, Cambridge CB3 0WA

E-mail address: c.sparber@damtp.cam.ac.uk 